\title{
Biased signaling downstream of epidermal growth factor receptor regulates proliferative versus apoptotic response to ligand
}

\author{
Remah Ali ${ }^{1}$, Wells Brown' ${ }^{1}$ Stephen Connor Purdy ${ }^{1}$, V. Jo Davisson $\mathbb{1}^{1,2}$ and Michael K. Wendt $\mathbb{0}^{1,2}$
}

\begin{abstract}
Inhibition of epidermal growth factor receptor (EGFR) signaling by small molecule kinase inhibitors and monoclonal antibodies has proven effective in the treatment of multiple cancers. In contrast, metastatic breast cancers (BC) derived from EGFR-expressing mammary tumors are inherently resistant to EGFR-targeted therapies. Mechanisms that contribute to this inherent resistance remain poorly defined. Here, we show that in contrast to primary tumors, ligandmediated activation of EGFR in metastatic BC is dominated by STAT1 signaling. This change in downstream signaling leads to apoptosis and growth inhibition in response to epidermal growth factor (EGF) in metastatic BC cells. Mechanistically, these changes in downstream signaling result from an increase in the internalized pool of EGFR in metastatic cells, increasing physical access to the nuclear pool of STAT1. Along these lines, an EGFR mutant that is defective in endocytosis is unable to elicit STAT1 phosphorylation and apoptosis. Additionally, inhibition of endosomal signaling using an EGFR inhibitor linked to a nuclear localization signal specifically prevents EGF-induced STAT1 phosphorylation and cell death, without affecting EGFR:ERK1/2 signaling. Pharmacologic blockade of ERK1/2 signaling through the use of the allosteric MEK1/2 inhibitor, trametinib, dramatically biases downstream EGFR signaling toward a STAT1-dominated event, resulting in enhanced EGF-induced apoptosis in metastatic BC cells. Importantly, combined administration of trametinib and EGF also facilitated an apoptotic switch in EGFR-transformed primary tumor cells, but not normal mammary epithelial cells. These studies reveal a fundamental distinction for EGFR function in metastatic BC. Furthermore, the data demonstrate that pharmacological biasing of EGFR signaling toward STAT1 activation is capable of revealing the apoptotic function of this critical pathway.
\end{abstract}

\section{Introduction}

Breast cancer metastasis is a multi-step process that culminates in vital organ invasion and proliferation by cancer cells. These later events of metastasis are responsible for patient morbidity and mortality in breast cancer ${ }^{1}$. Developing targeted therapies for metastatic breast cancer faces many challenges. Paramount to these challenges is

\footnotetext{
Correspondence: Michael K. Wendt (mwendt@purdue.edu)

${ }^{1}$ Department of Medicinal Chemistry and Molecular Pharmacology, Purdue University, West Lafayette, IN 47907, USA

${ }^{2}$ Purdue University Center for Cancer Research, West Lafayette, IN 47907, USA Edited by M. Agostini
}

the high degree of molecular changes that characterize metastatic lesions compared to primary tumors, which constantly brings into question the utility of primary tumor analysis to guide metastatic therapy ${ }^{2,3}$. Thus, understanding signaling events specific to metastatic breast tumors is essential to identify potential therapeutic targets and biomarkers for late-stage disease.

Similar to more established breast cancer-associated genes, such as estrogen receptor (ER) and human epidermal growth factor receptor 2 (Her2), primary versus metastatic tumor discrepancies have also been described for epidermal growth factor receptor (EGFR)-expressing

\section{(c) The Author(s) 2018}

(c) (i) Open Access This article is licensed under a Creative Commons Attribution 4.0 International License, which permits use, sharing, adaptation, distribution and reproduction c. in any medium or format, as long as you give appropriate credit to the original author(s) and the source, provide a link to the Creative Commons license, and indicate if changes were made. The images or other third party material in this article are included in the article's Creative Commons license, unless indicated otherwise in a credit line to the material. If material is not included in the article's Creative Commons license and your intended use is not permitted by statutory regulation or exceeds the permitted use, you will need to obtain permission directly from the copyright holder. To view a copy of this license, visit http://creativecommons.org/licenses/by/4.0/. 
mammary tumors ${ }^{3-6}$. Breast cancer cells predominantly respond to EGFR agonists in a proliferative fashion supporting its role as an oncogene. Indeed, studies from our group and others have linked activation of EGFR to mammary epithelial cell transformation, increased proliferation, and several early steps of metastasis ${ }^{7,8}$. Various signaling pathways facilitate these oncogenic roles of EGFR, including the p38 mitogen-activated protein kinase, extracellular signal-regulated kinases 1 and 2 (ERK1/2), signal transducer and activator of transcription 3 (STAT3), and phosphoinositide 3-kinase (PI3K). These experimental findings are supported by clinical studies that report high expression of EGFR in primary mammary tumors is predictive for reduced patient survival ${ }^{9,10}$.

However, subsets of cancer cells, including those originating from the breast, respond to epidermal growth factor (EGF) via cell cycle arrest and induction of apoptosis $^{11-14}$. These observations are corroborated by the antitumor response of in vivo administered $\mathrm{EGF}^{12}$. Many studies describe the growth-inhibitory functions of EGFR to be mediated by STAT1, which is an established tumor suppressor and mediator of apoptosis downstream of interferon signaling ${ }^{15-17}$. We have recently shown that EGFR function changes from oncogenic in primary tumors to growth-inhibitory and apoptotic in metastatic tumors ${ }^{5,13}$. The importance of this paradoxical function of EGFR is substantiated by the failure of EGFR inhibition (EGFRi) to improve the clinical outcomes of metastatic breast cancer patients ${ }^{18-26}$. Inhibition of specific pathways downstream of EGFR is also being pursued for clinical applications. In particular, the compound trametinib is an allosteric inhibitor of MEK1/2, the kinases directly upstream of ERK1/2 $2^{27}$. As opposed to direct inhibition of growth factor receptors, targeting of downstream pathways requires consideration that the cellular effects of inhibition may also arise via differential activation of alternate signaling pathways downstream of a common driver receptor.

In the current study, we demonstrate the apoptotic function of EGFR in metastatic breast cancer is dependent on STAT1 and we address the hypothesis that pharmacologic inhibition of MEK $1 / 2$ with trametinib will bias EGFR signaling toward a STAT1-dominated, apoptotic signaling pathway. These findings identify unique molecular signaling events that specifically manifest in metastatic BC, and identify a pharmacological approach to enhance STAT1-induced apoptosis and limit primary and metastatic tumor growth.

\section{Methods and materials Cell lines and reagents}

Murine NMuMG and human MDA-MB-468 cells were purchased from ATCC and cultured in DMEM containing $10 \%$ or $5 \%$ FBS, respectively. MDA-MB- 468 passages
1-5 were used in this study. NMuMG cells and their metastatic variants also received $10 \mu \mathrm{g} / \mathrm{ml}$ of insulin. Construction of NMuMG cells expressing human mutant (EGFR-AA) or WT-EGFR (NME) and their metastatic variants are described elsewhere ${ }^{7,28}$. Cellular depletion of STAT1 cells was accomplished by VSVG lentiviral transduction of pLKO.1 shRNA vectors (Thermo Scientific), sequences of shRNAs can be found in Supplementary Table 1 . The human MCF10-Ca1a cell line was kindly provided by Dr. Fred Miller (Wayne State University) and cultured in DMEM supplemented with 10\% FBS. A list of the chemical inhibitors and growth factors used throughout the study can be found in Supplementary Table 2.

\section{Immunoblot and immunofluorescent analyses}

For immunoblot assays, equal aliquots of nuclear, cytoplasmic, or whole-cell lysates were resolved by SDSPAGE and transferred to PVDF membranes using standard methods. Nuclear and cytoplasmic fractions were generated via a differential lysis using Buffer A $(10 \mathrm{mM}$ HEPES; pH 7.9, $10 \mathrm{mM} \mathrm{KCL,} 0.1 \mathrm{mM}$ EDTA, and protease and phosphatase inhibitors) to obtain a cytoplasmic fraction. Intact nuclei were pelleted from these lysates and washed twice in fresh Buffer A before being lysed in Buffer B (20 mM HEPES; pH 7.9, $0.4 \mathrm{M} \mathrm{NaCl}, 1 \mathrm{mM}$ EDTA, $10 \%$ glycerol, and protease and phosphatase inhibitors). Immunofluorescent assays were conducted using primary antibodies in combination with fluorescently labeled secondary antibodies. Confocal images were captured using a Nikon A1Rsi inverted microscope. Super-resolution images are structure illuminations obtained on a Nikon Ti-E inverted microscope with $\mathrm{N}$ SIM capability. Antibody concentrations and suppliers are listed in Supplementary Table 3.

\section{Apoptosis and cell viability assays}

Caspase 3/7 activity was quantified using the CaspaseGlo 3/7 assay (Promega) according to the manufacturer's instructions. Cell viability was measured using the CellTiter-Glo assay (Promega) according to the manufacturer's instructions. Floating cells were collected from all conditions, spun down, resuspended in trypsin to break clumps of cells into single-cell suspension. For Annexin V staining, adherent cells were trypsinized into single-cell suspensions and added to the floating cell fraction and analyzed for Annexin V/PI staining using a FITC Apoptosis Detection Kit I (BD Pharmingen).

\section{D culture methods}

Primary and metastatic breast cancer cells $\left(5 \times 10^{3}\right)$ were plated into non-adherent round bottom 96-well plates (Corning) in full growth media and cultured for 3 days. At this point, the tumorspheres were physically 
transferred with $50 \mu \mathrm{l}$ of residual media and $150 \mu \mathrm{l}$ of fresh media to a flat bottom 96-well plate coated with $50 \mu \mathrm{l}$ of growth factor reduced basement membrane hydrogel (Trevigen) in the presence or absence of trametinib and EGF. These structures were allowed to grow for an additional 3 days for metastatic NM-LM1 cells or 10 days for non-metastatic NME cells at which point structure size was quantified using image $J$ analyses or invasive tips were enumerated.

\section{Statistical methods}

Statistical analyses were carried out using unpaired Student's $T$-test or ANOVA where the data fit the parameters of the test. $P$ values $<0.05$ were considered statistically significant. $P$ values for all experiments are indicated. All $P$ values were generated using PrismGraphPad software.

\section{Results}

\section{EGF-mediated STAT1 phosphorylation increases with} metastasis

Our previous studies demonstrate that EGFR overexpression is capable of transforming normal murine mammary gland (NME) cells $s^{7,13,29,30}$. Transient induction of epithelial-mesenchymal-transition (EMT) via treatment with TGF- $\beta$ facilitates the metastasis and inherent resistance of these cells to EGFRi (Fig. 1a) ${ }^{13}$. To investigate differential downstream signals generated by EGFR in these isogeneic cells of increasing metastatic capacity, we examined the phosphorylation of STAT1 and ERK1/2 in response to exogenous EGF stimulation. As shown in Fig. 1b, c, EGF treatment resulted in enhanced phosphorylation of STAT1 in lung-metastatic, LM1 and LM2, cells as compared to the nonmetastatic NME cells. In contrast, phosphorylation of ERK1/2 in response to EGF was similar in all cell types, therefore the ratio of ERK $1 / 2$ to STAT1 phosphorylation is dramatically altered in metastatic cells (Fig. 1b, c). Importantly, the enhanced STAT1 signaling in the LM cells occurs despite EGFR returning to levels similar to endogenous in nontransformed NMuMG cells (Fig. 1b). Unlike EGF, interleukin 6 (IL6)-induced STAT3 activation was similar across all cells of the NME series (data not shown). Taken together, these findings suggest that through metastasis there is not a general propensity to increase STAT activation, but there is a specific increase in the EGFR: STAT1 signaling axis.

To expand these observations, we derived additional metastatic lines from different anatomical locations. Metastases from two different lymph nodes were subcultured and termed NME-Lym1 and NME-Lym2 cell lines (Fig. 1d). Consistent with our previously reported observations in lung metastases, these lymph node metastases display increased resistance to the EGFR inhibitor erlotinib as compared to primary tumor NME cells when cultured under 3D organotypic conditions (Fig. S1). Furthermore, the downstream EGFR signaling in these lymph node metastases also became dominated by STAT1 phosphorylation (Fig. 1e). The diminution of EGFR through metastasis is further supported by previous reports from our lab and others showing EGFR downregulation in the RAStransformed MCF10A progression series ${ }^{6,13,31}$. To examine EGF-induced downstream signaling in this additional isogenic model of metastatic progression, we engineered the metastatic Ca1a cells to re-express EGFR using stable or doxycycline-inducible expression systems (Figs. If and S2). EGF stimulation of Cala cells stably or transiently replenished with EGFR expression led to robust phosphorylation of EGFR and STAT1 and induction of apoptosis (Figs. If and S2). Overall, these data indicate that enhanced STAT1 signaling downstream of EGFR activation correlates with EGF-mediated growth inhibition in metastatic BC.

\section{STAT1 is required for EGF-mediated apoptosis in metastatic BC}

Previous studies indicate that STAT1 activation by EGF or other cytokines inhibits proliferation and induces apoptosis $^{15-17}$. Therefore, we sought to define the functional role of STAT1 downstream of EGFR activation in metastatic breast cancer cells. Indeed, EGF stimulation of both cell lines derived from lymph node metastases, Lym1 and Lym2, resulted in cell rounding and enhanced caspase 3/7 activity (Fig. 2a, b). Depletion of STAT1 expression in the metastatic Lym1 cells using two different shRNA sequences prevented the ability of EGF to induce apoptosis (Fig. 2c, d). Identical results were observed when STAT1 was depleted in the Lym2 cell line (data not shown). In these analyses, we also pharmacologically blocked Erk1/2 signaling through the addition of trametinib. While the addition of trametinib alone had no effect on apoptosis, it did potentiate the ability of EGF to induce apoptosis in these cells (Fig. 2d). Importantly, this effect was similarly dependent on STAT1 expression (Fig. 2d) These data clearly implicate the functional involvement of STAT1 in EGF-induced apoptosis in metastatic BC.

\section{Nuclear STAT1 is accessed by EGFR through endocytosis}

Our recent studies demonstrate an enhanced localization of EGFR in the nucleus of metastatic breast cancer cells as compared to primary tumor cells ${ }^{32}$. Given the role of STAT1 in facilitating EGF-induced apoptosis in metastatic cells, we next sought to investigate the subcellular localization of STAT1 under nonstimulated and EGF-stimulated conditions. Surprisingly, STAT1 was already localized to the nucleus in NME cells even before ligand stimulation (Figs. 3a and S3). Unfortunately, immunostaining these cells with a phospho-specific STAT1 antibody is not possible due to cross-reactivity 


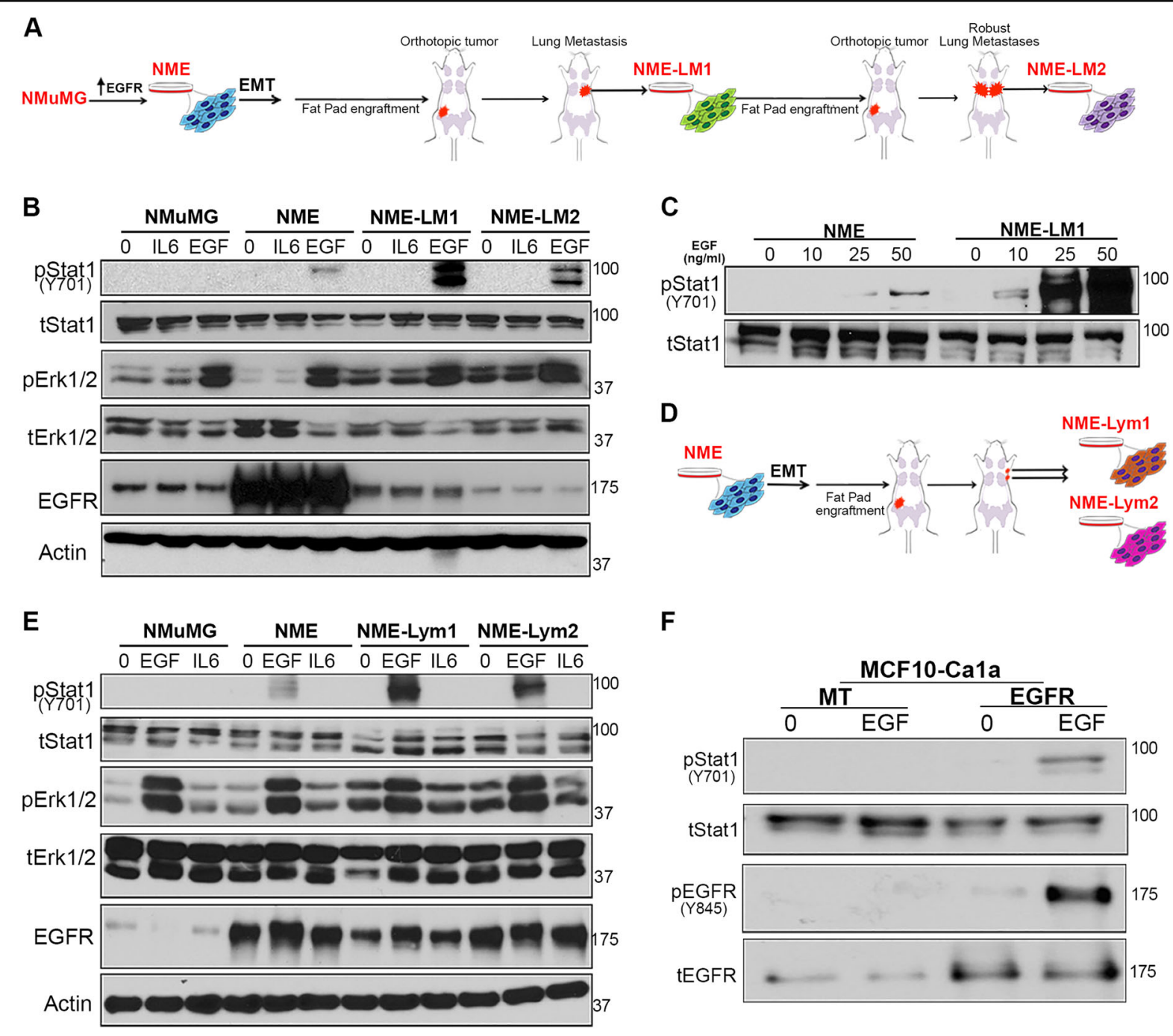

Fig. 1 The STAT1:ERK1/2 signaling ratio downstream of EGFR is altered in metastatic cells compared to primary breast cancer cells. a Schematic representation of the EGFR-transformed lung-metastatic breast cancer progression series. $\mathbf{b}$ Cells described in panel a were analyzed by immunoblot for phosphorylation of STAT1 and ERK1/2 in response to a $30 \mathrm{~min}$ EGF stimulation ( $50 \mathrm{ng} / \mathrm{ml}$ ). IL6 and BSA (0) served as protein stimulation controls, and analysis of total levels of EGFR, STAT1, ERK1/2, and Actin served as loading controls. c Non-metastatic (NME) and lung metastatic (NME-LM1) cells as described in panel a were stimulated for 30 min with the indicated concentrations of EGF and analyzed for phosphorylation of STAT1. Analysis of total STAT1 served as a loading control. d Schematic representation of the EGFR-transformed lymph nodemetastatic breast cancer progression series. Metastatic cells were isolated from two separate lymph-nodes and termed NME-Lym1 and NME-Lym2. e Cells described in panel $\mathbf{d}$ were analyzed for phosphorylation of STAT1 and ERK1/2 in response to a $30 \mathrm{~min}$ EGF stimulation (50 $\mathrm{ng} / \mathrm{ml})$. IL6 and BSA served as stimulation controls, and analysis of total levels of EGFR, STAT1, ERK1/2, and Actin served as loading controls. $\mathbf{f}$ EGFR was ectopically expressed in metastatic MCF10-Ca1a cells. These cells were stimulated with EGF (50 ng/ml) for $30 \mathrm{~min}$ and analyzed for phosphorylation of STAT1 and EGFR. Analysis of total levels of EGFR and STAT1 served as loading controls. All immunoblots shown are representative of at least three independent experiments

with phospho-EGFR epitopes. However, our whole-cell and nuclear-fractioned immunoblot analyses indicate that prior to EGF stimulation, STAT1 is not phosphorylated (Figs. 1b and S3). Therefore, these data are consistent with previous reports that indicate a pool of STAT1 can exist in the nucleus in an unphosphorylated state ${ }^{33}$. In contrast, ERK $1 / 2$ is primarily localized in the cytoplasm and only moves into the nucleus upon EGF stimulation, an event that is stabilized upon the addition of leptomycin B to prevent nuclear export (Fig. 3b). These data suggest that EGFR must gain access to the nucleus to phosphorylate STAT1. Indeed, EGFR internalization in endocytic vesicles can clearly be visualized upon EGF stimulation (Fig. 3a). Moreover, a closer examination of EGFR localization using super-resolution microscopy revealed that in certain areas of the cell the plasma membrane is in 

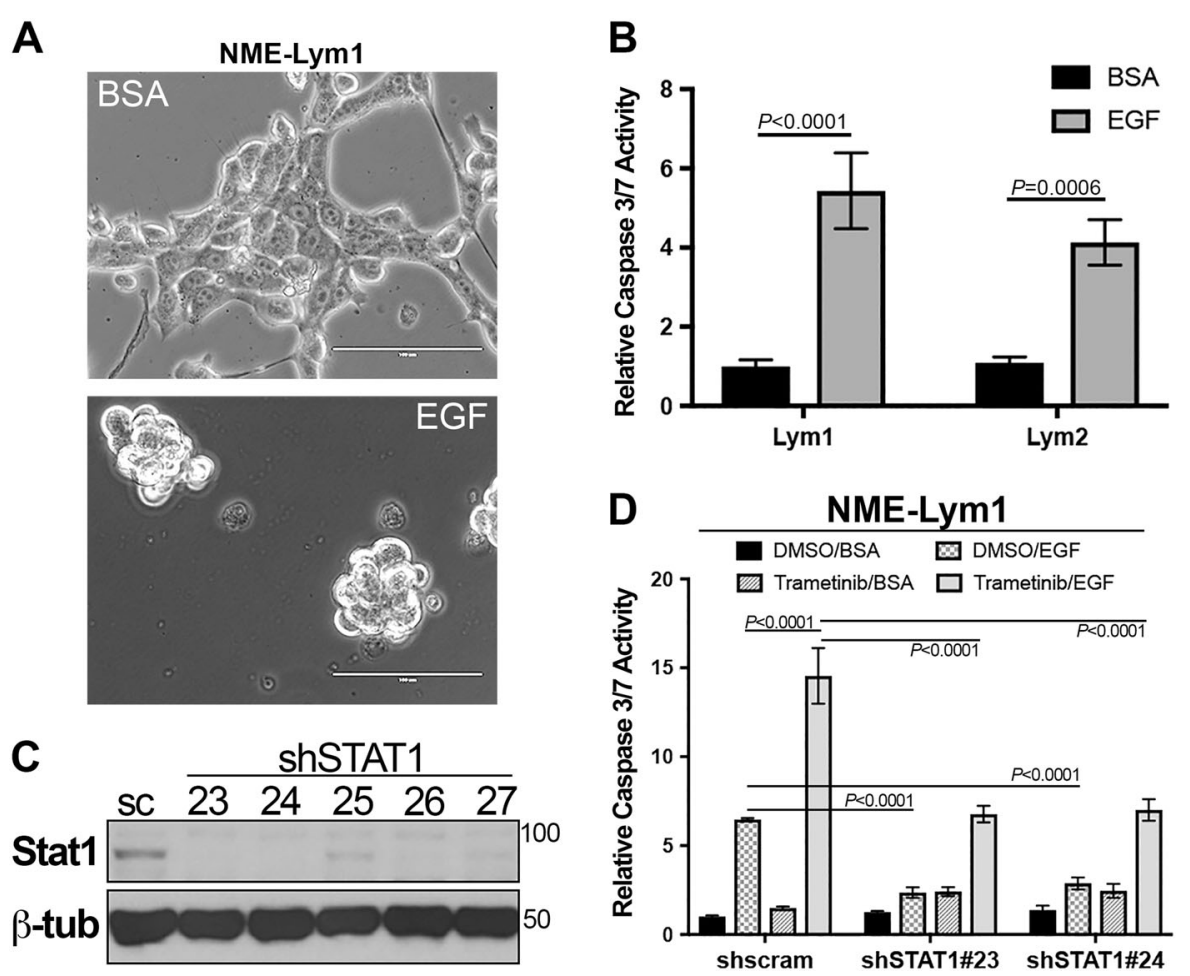

Fig. 2 STAT1 is required for EGF-induced apoptosis in metastatic breast cancer cells. a Lymph node metastases (NME-Lym1 and NME-Lym2) were stimulated with EGF $(100 \mathrm{ng} / \mathrm{ml})$ for $36 \mathrm{~h}$ and imaged via phase contrast microscopy. b Following EGF stimulation as described in panel a, cells were assayed for caspase 3/7 activity. c NME-Lym1 cells were constructed to stably express a scrambled control (sc) shRNA or various shRNAs (23-27) targeting STAT1. These cells were analyzed for STAT1 expression by immunoblot. Expression of $\beta$-tubulin ( $\beta$-tub) served as a loading control. d Control (shscram) and STAT1 depleted (shSTAT1\#23 and shSTAT1\#24) NME-Lym1 cells were treated with EGF as described above and caspase 3/7 activity was assessed. Separate groups of cells were treated with the MEK1/2 inhibitor trametinib $(100 \mathrm{nM})$ in the presence or absence of exogenous EGF $(100 \mathrm{ng} / \mathrm{ml})$ and these cells were similarly assayed for caspase $3 / 7$ activity. Data in panels $\mathbf{b}$ and $\mathbf{c}$ are the mean \pm SE of three independent experiments completed in triplicate resulting in the indicated $P$ values

direct physical contact with the nucleus. Therefore, we hypothesized that upon ligand-induced endocytosis from the plasma membrane, a subpopulation of EGFR molecules has immediate access to the nuclear compartment (Fig. 4a). To this end, we utilized an EGFR construct that contains alanine substitutions in the juxtamembrane dilysine motif (679-680-LL converted to AA). This construct is established to be signaling proficient from the plasma membrane, but deficient in endocytosis upon ligand engagement ${ }^{34}$. Accordingly, the EGFR-AA construct was not able to induce phosphorylation of STAT1 in response to EGF (Fig. 4b). Taken together, these data consistently indicate that a subset of EGF receptors is able to translocate to the nucleus via endocytosis to phosphorylate STAT1.

\section{Pharmacological biasing of EGFR signaling promotes} EGF-induced apoptosis in metastatic breast cancer cells

To specifically target the function of the subpopulation of EGF receptors that reach the nuclear compartment, we utilized our recently reported novel EGFR inhibitor ${ }^{32}$.
This chemical construct contains the EGFR tyrosine kinase inhibitor gefitinib linked to a peptoid moiety encoding the SV40 nuclear localization sequence (NLSgefitinib). This approach leads to robust accumulation of geftinib in the nucleus ${ }^{32}$. Consistent with the notion that only endocytosed EGFR molecules have access to the nuclear pool of STAT1, pretreatment of NME-LM1 cells with this chimeric NLS-gefitinib molecule led to potent blockade of EGF-induced STAT1 phosphorylation without affecting phosphorylation of ERK1/2 (Fig. 5a). In contrast, pretreatment with trametinib drastically alters the STAT1:ERK1/2 activation ratio by completely preventing downstream phosphorylation of ERK1/2, while leading to a slight increase in EGF-induced phosphorylation of STAT1 (Fig. 5a).

To quantify the biological implications of these events, we again analyzed caspase 3/7 activation following EGF treatment in the presence or absence of these inhibitors (Fig. 5b). Consistent with our findings in Fig. 2 implicating the role of STAT1 in facilitating EGF-induced apoptosis, the addition of gefitinib-NLS blocked EGF-induced 

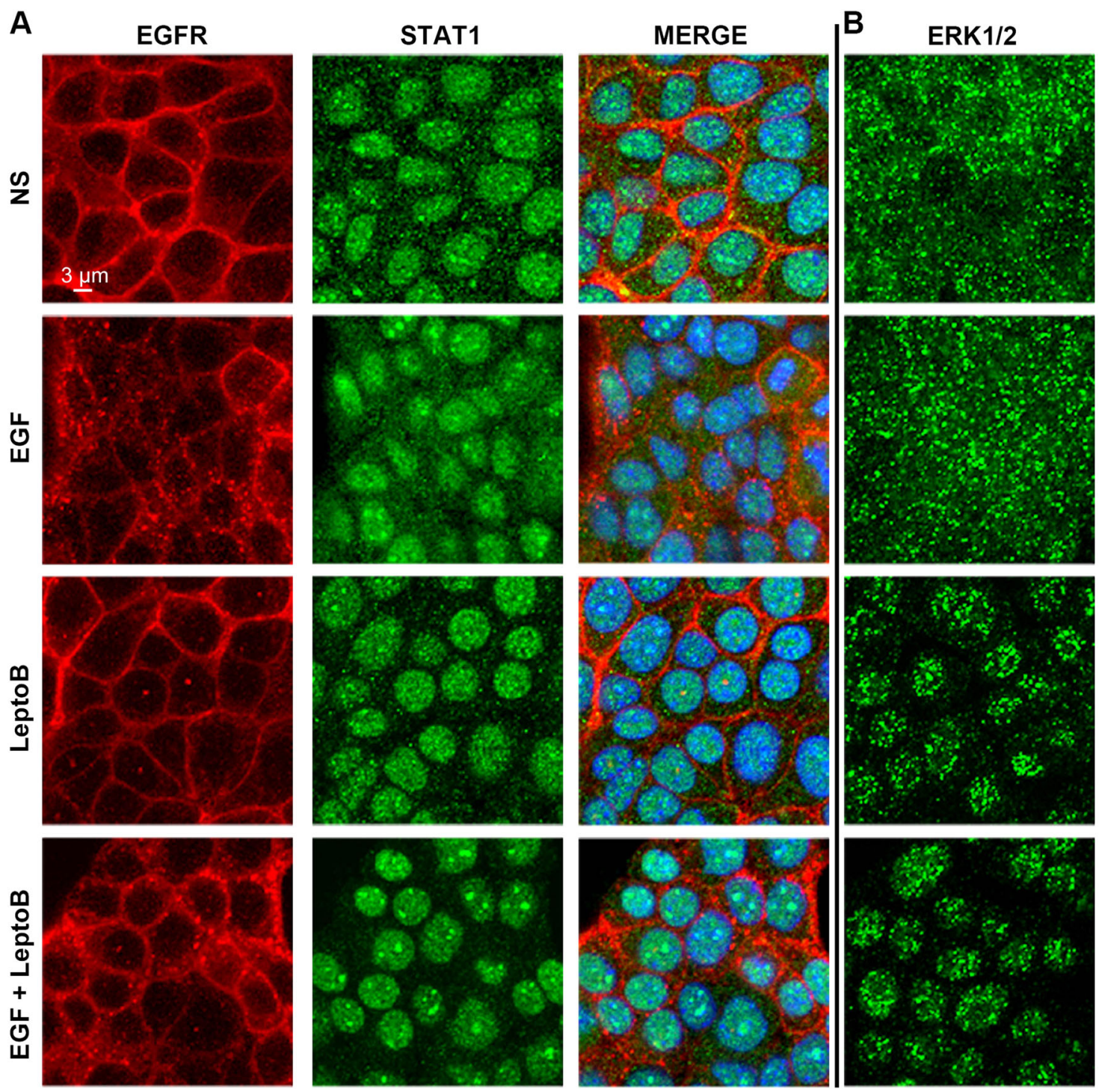

Fig. 3 STAT1 is localized in the nucleus prior to phosphorylation. a NME cells were stimulated with EGF for 30 min in the presence or absence of the nuclear export inhibitor leptomycin B (LeptoB). These cells were subsequently analyzed by dual immunofluorescence and imaged via confocal microscopy for the localization of EGFR and STAT1. These cells were counterstained with DAPI to visualize the nuclei. $\mathbf{b}$ Separate sets of cells were stimulated as in panel $\mathbf{a}$ and analyzed for localization of ERK1/2. Data in panels $\mathbf{a}$ and $\mathbf{b}$ are representative images from at least 10 fields of view over two independent experiments

activation of caspase $3 / 7$ (Fig. 5b). Consistent with the induction of apoptosis, cotreatment of these lung metastases with trametinib led to a robust increase in EGFinduced caspase 3/7 activity and Annexin V staining (Figs. 5b and S4). Also, NLS-gefitinib was capable of preventing EGF-mediated growth inhibition in the NMELM1 cells, and NLS-gefitinib increased cell viability under nonstimulated conditions (Fig. $5 \mathrm{c}$ ). In contrast, the addition of EGF augmented the growth suppressive effects of trametinib in a shorter-term assay (Fig. 5d). Consistent with our shRNA depletion studies, the ability of EGF to induce apoptosis is likely dependent on STAT1 since the addition of IL-6, a specific activator of STAT3, did not alter trametinib-induced growth inhibition (Fig. 5d). All of these events could be replicated using the LM2 metastatic variant (Fig. S5). The only noted difference in the LM2 cells was that no caspase $3 / 7$ activity could be quantified with EGF alone, but again the addition of EGF and trametinib led to significantly increased apoptosis as compared to trametinib alone (Fig. S5a).

As cells escape primary mammary tumors, a particularly aggressive subpopulation is able to survive the nonadherent conditions in blood/lymphatic circulation, and these cells are ultimately responsible for colonizing vital 


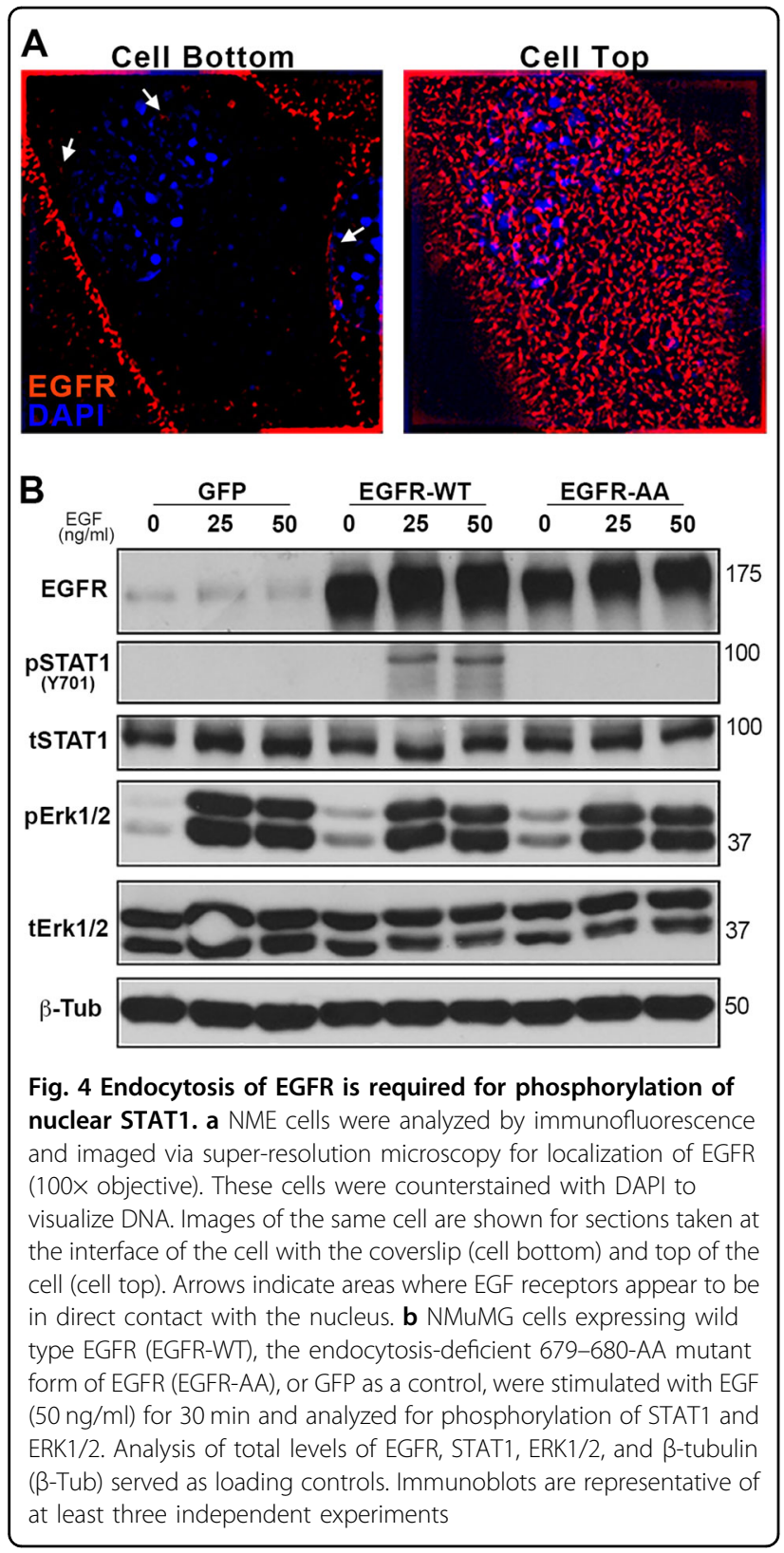

organs. To recapitulate these events in an in vitro assay, we generated spheroids of metastatic cells in nonadherent conditions and then transferred these spheroids onto a bed of reconstituted basement membrane (Fig. 5e). Using this approach, the metastatic NME-LM1 tumorspheres form highly invasive, multicellular branches over a period of 3 days (Fig. 5e). The addition of physiological amounts of EGF or nanomolar concentrations of trametinib partially blocked these events, but a combination of the growth factor and trametinib completely prevented the invasive growth of these highly metastatic cells. Therefore, biasing EGFR signaling toward STAT1 using downstream inhibitors can enhance the apoptotic potential of this pathway.

Pharmacological biasing of EGFR signaling fundamentally changes response to EGF in primary tumor cells

We next sought to determine if biasing EGFR signaling could change the EGF response of primary tumor cells from proliferative to apoptotic. Therefore, we treated the nonmetastatic NME cells with EGF in the presence of NLS-gefitinib, unconjugated gefitinib, or trametinib. As observed in Fig. 1, very little activation of STAT1 in NME cells occurred upon EGF stimulation, however this pathway was greatly enhanced upon inhibition of MEK $1 / 2$ with trametinib (Fig. 6a). Consistent with the notion that the ERK1/2:STAT1 activation ratio dictates the proliferative versus apoptotic outcome of EGF, we observed a drastic induction of apoptosis in the NME cells upon coadministration of EGF and trametinib, whereas either treatment alone did not produce any caspase activation (Fig. 6b, c). Moreover, these effects could not be produced in control cells expressing normal amounts of EGFR, or in cells expressing the EGFR-AA variant that is deficient in STAT1 activation (Fig. S6 and Fig. 6c). Finally, using our 3D spheroid assay described in Fig. 5 we could illustrate the non-metastatic nature of the NME cells as they fail to form any invasive structures (Fig. 6d). However, we could quantify an increase in spheroid size with the addition of exogenous EGF, a result that was completely prevented upon addition of trametinib (Fig. 6d, e). Moreover, cotreatment with EGF and trametinib lead to the appearance of apoptotic bodies around the tumorspheres (Fig. 6d; inset). These data indicate that biasing signaling away from ERK1/2 can fundamentally alter how cells respond to ligand-mediated activation of EGFR.

\section{EGFR:STAT1 signaling augments trametinib-induced growth inhibition}

To extend our findings to other breast cancer models of EGFR signaling, we applied a similar EGF-trametinib treatment combination to our dox-inducible model of EGFR expression in the metastatic Ca1a cells. When EGFR expression was induced with dox, we observed STAT1 phosphorylation in response to EGF stimulation (Fig. 7a). Consistent with the Cala cells being transformed by a constitutively active form of RAS, we did not observe any further ERK1/2 phosphorylation upon EGF stimulation (Fig. 7a). However, EGF-induced phosphorylation of STAT1 was enhanced in the presence of trametinib concentrations capable of completely blocking this constitutive phosphorylation of ERK1/2 (Fig. 7a). Using these cells, we observed a dose-dependent induction of EGFR expression with dox (Fig. 7b). This transient induction resulted in an EGFR-dependent inhibition of cell growth in the presence of EGF and trametinib that was not 


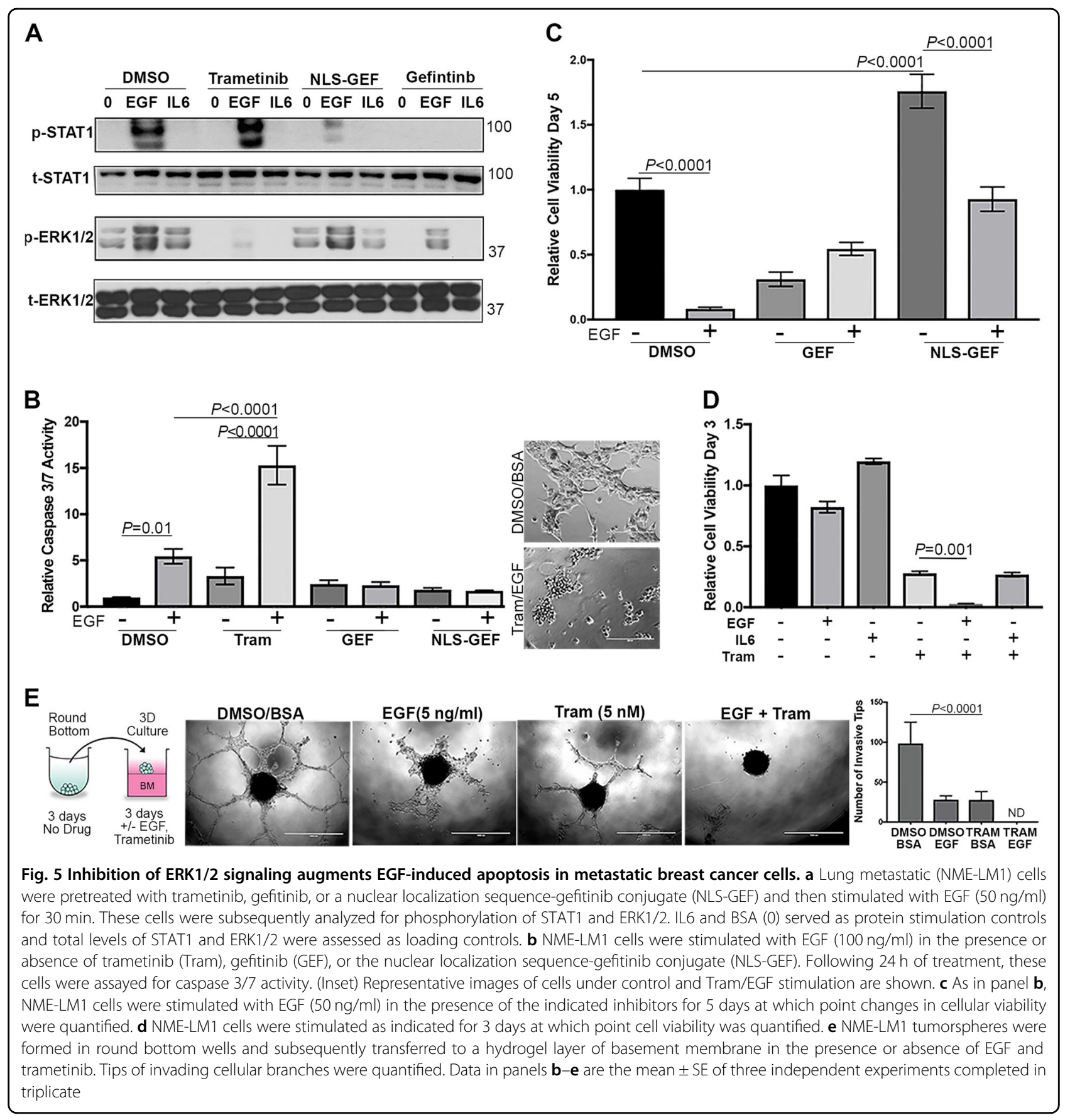

observed in the presence of trametinib alone (Fig. 7c). We similarly assessed the MDA-MB-468 (468) model of triple negative breast cancer. As we observed in our metastatic cells and consistent with previous reports, treatment of the 468 cells with EGF induced phosphorylation of STAT1 (Fig. 7d) ${ }^{16}$. EGF-induced phosphorylation of STAT1 was again completely blocked by our nuclearlocalized EGFR inhibitor, a condition that has no effect on ERK1/2 phosphorylation (Fig. 7d). Importantly, pretreatment with trametinib at a concentration that completely prevented ERK1/2 phosphorylation enhanced EGF-induced STAT1 signaling (Fig. 7d). Finally, combined treatment with EGF and trametinib lead to a potent inhibition of cell viability as compared to either treatment alone (Fig. 7e). Such a combination effect was not observed with IL6 which has been shown to inhibit the growth of 468 cells by conferring stem-like properties but is unable to induce STAT1 phosphorylation. 


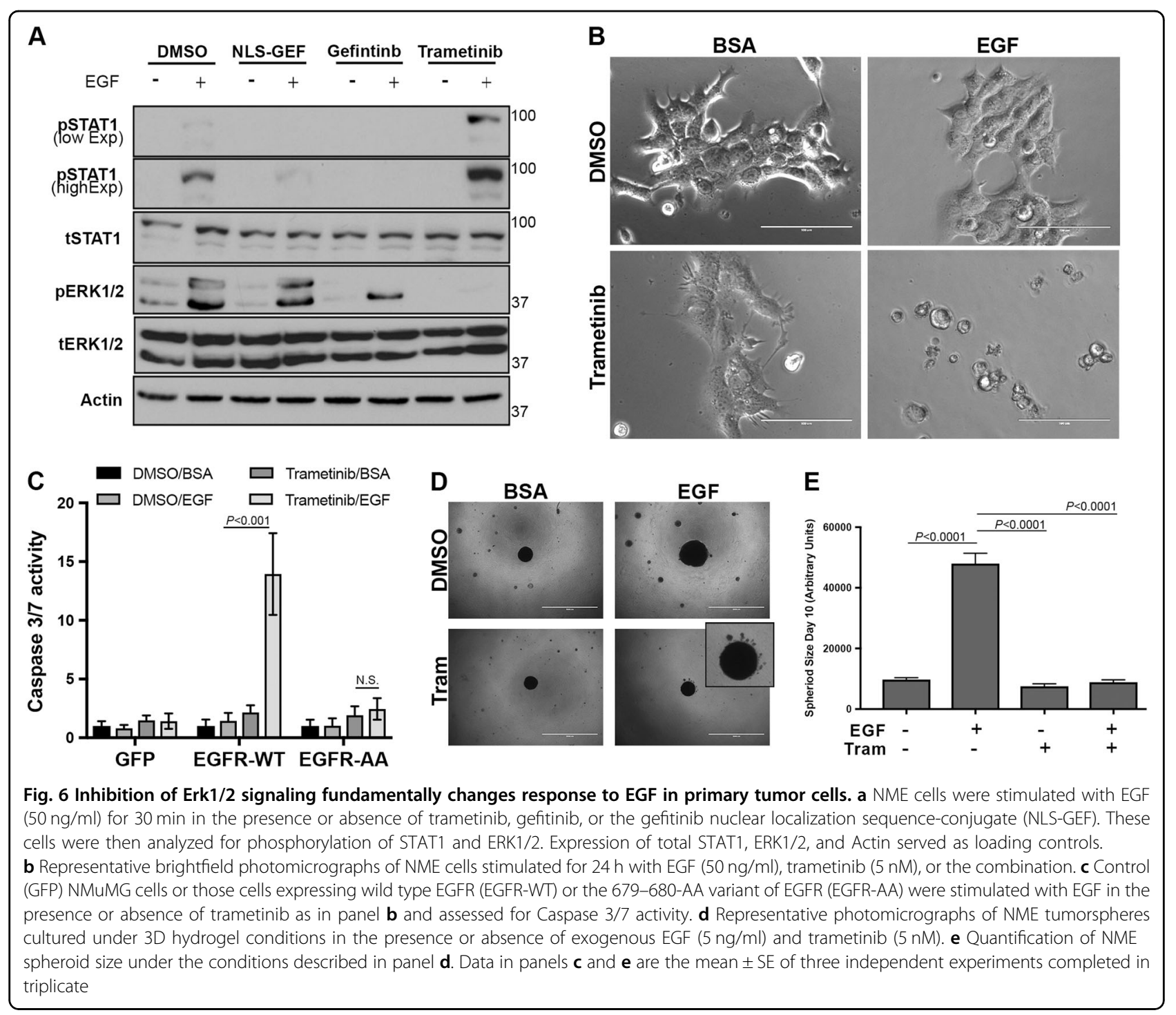

\section{Discussion}

EGFR activation is upstream of multiple signal transduction pathways. The differential activation of particular pathways in response to ligand leads to oncogenic versus apoptotic signals in specific cell types ${ }^{5,35,36}$ (Fig. 8). We have previously reported that EGFR function paradoxically changes from oncogenic to apoptotic after in vivo metastasis of $\mathrm{BC}$ cells ${ }^{13}$. In the current study, we demonstrate that ligand-mediated EGFR activation ultimately results in a nuclear STAT1-dependent apoptosis of metastatic $\mathrm{BC}$ cells. This fundamental change in response to EGF through breast cancer progression led us to address the hypothesis that pharmacological biasing of downstream signaling could reveal apoptotic EGFR signaling, even in early-stage breast cancer. This concept is supported by recent studies in the fields of G-protein coupled receptor signaling and receptor tyrosine kinase signaling, which indicate that differential ligand stimulation leads to biased downstream pathway activation and therefore unique biological outputs ${ }^{37,38}$. These previous studies have focused on unnatural ligands and allosteric modulation of receptors. In contrast, our work herein demonstrates that unique cellular outcomes in response to an endogenous ligand can be manifested when specific downstream pathways are pharmacologically interdicted.

Recent studies indicate that constitutive EGFR signaling induced upon receptor mutations are distinct and mutually exclusive as compared to ligand-induced signaling ${ }^{39}$. Our findings indicate that direct pharmacological targeting of WT-EGFR using our nuclear-localized gefitinib conjugate completely prevents STAT1 activation, leading to enhanced cell growth as this compound has no effect on the ability of EGFR to signal to ERK1/2. Furthermore, in several instances, we observed unconjugated gefitinib prevents STAT1-mediated apoptotic signaling 

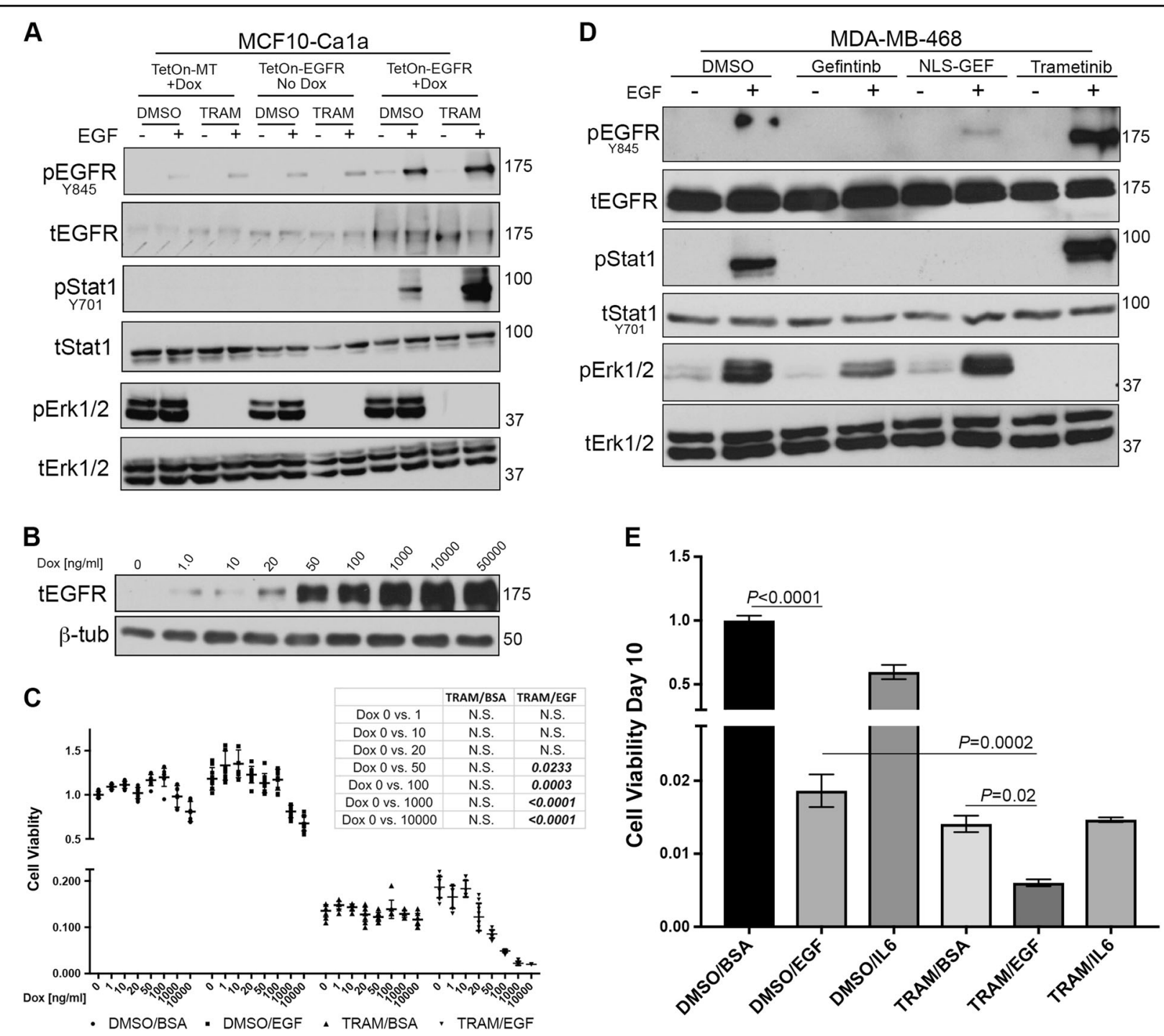

Fig. 7 EGF enhances the growth inhibitory effect of trametinib. a Metastatic MCF10-Ca1a cells were constructed to express a control (TetOn-MT) or EGFR (TetOn-EGFR) encoding vector under the control of doxycycline (Dox) inducible promoter. These cells were pretreated with Dox for $24 \mathrm{~h}$ and subsequently stimulated with EGF $(50 \mathrm{ng} / \mathrm{ml})$ for $30 \mathrm{~min}$ in the presence or absence of trametinib (TRAM). Phosphorylation of EGFR, STAT1, and ERK1/ 2 was assessed. Expression of total EGFR, STAT1, and ERK1/2 served as loading controls. b MCF10-Ca1a TetOn-EGFR cells were stimulated with the indicated concentrations of Dox for $24 \mathrm{~h}$ and assessed for expression of EGFR. $\beta$-Tub served as a loading control. c MCF10-Ca1a TetOn-EGFR cells were stimulated with the indicated concentrations of Dox for 10 days in the presence or absence of EGF and trametinib and cell viability was quantified. Chart inset: The resultant $P$ values of ANOVA analyses comparing the indicated treatment groups under control (Dox 0) and Dox conditions. d The MDA-MB-468 cells were stimulated with EGF $(50 \mathrm{ng} / \mathrm{ml})$ for $30 \mathrm{~min}$ in the presence or absence of trametinib and assessed for phosphorylation of EGFR, STAT1, and ERK1/2. Expression of total EGFR, STAT1, and ERK1/2 served as loading controls. e The MDA-MB-468 cells were grown for a period of 10 days in the presence or absence of EGF $(50 \mathrm{ng} / \mathrm{ml})$, IL6 $(20 \mathrm{ng} / \mathrm{ml})$, trametinib $(5 \mathrm{nM})$ of the indicated combinations at which point cell viability was quantified. The indicated groups were analyzed by $T$-test resulting in the indicated $P$ values. Data in panels $\mathbf{a}$, $\mathbf{b}$, and $\mathbf{d}$ are representative of three independent analyses, and data in panels $\mathbf{c}$ and $\mathbf{e}$ are the mean \pm SE for three independent experiments completed in triplicate

downstream of ligand-activated WT-EGFR at a much lower concentration than is required for inhibition of ERK1/2-mediated proliferative signaling. These data suggest that complete to near-complete blockade of EGFR function is required before an anti-tumorigenic response would be expected in breast cancer cells bearing high levels of WT-EGFR undergoing ligand-mediated signaling. Indeed, complete pharmacological blockade of a target molecule is challenging if not impossible to achieve in vivo. This concept that incomplete inhibition of 


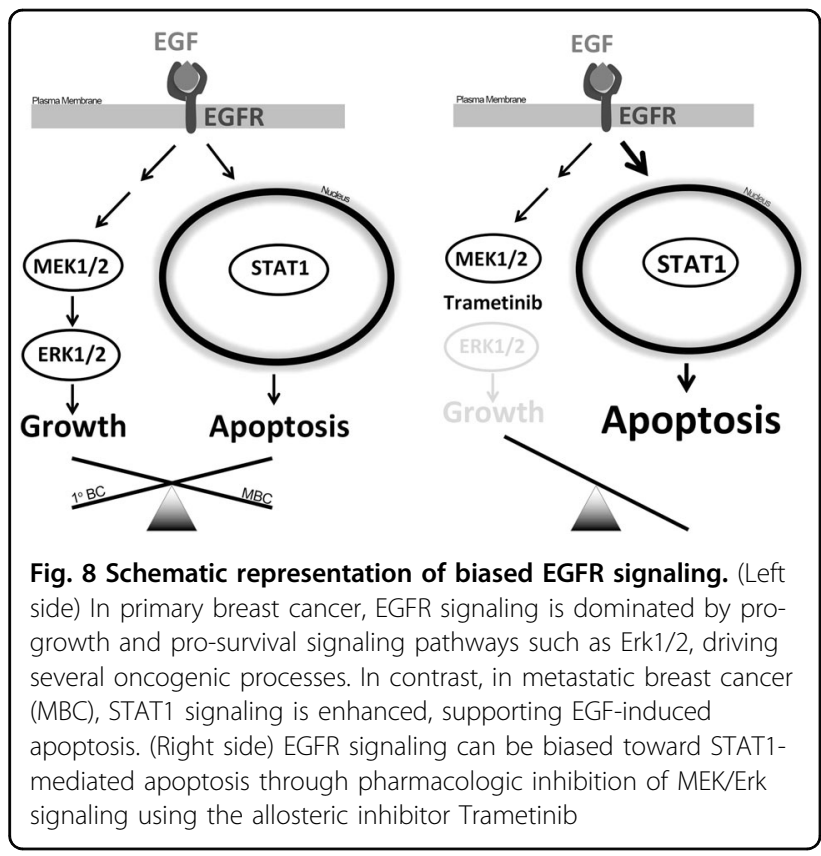

WT-EGFR is biasing signaling toward proliferative ERK1/ 2 signaling is completely consistent with the clinical failure of EGFR inhibitors in metastatic breast cancer ${ }^{18}$.

Our recent studies demonstrate that metastatic cells increase their nuclear pool of EGFR ${ }^{32}$. These data are consistent with the findings here demonstrating an enhanced ability of metastatic cells to access and phosphorylate nuclear STAT1 in response to EGF stimulation. The potential mechanisms by which metastatic cells increase their nuclear pool of EGFR are potentially numerous $^{39-41}$. However, recent data suggest that more migratory cells undergo constant nuclear rupture, and these cells repair these events by using components of endosomal sorting complexes ${ }^{42,43}$. Together with our findings using the EGFR-AA construct, these data suggest a mechanism in which more migratory and metastatic breast cancer cells undergo an increased rate of nuclear rupture and repair and thus sample more activated EGFR molecules from endosomes. These events would lead to an increased pool of nuclear EGFR and enhanced interaction of these receptors with the nuclear pool of STAT1. Finally, several of the model systems interrogated herein demonstrate that trametinib enhances EGFR: STAT1 signaling. Our recent studies indicate that EGFR signaling is regulated in metastatic cells via expression of the EGFR inhibitory molecule Mig6 ${ }^{13}$. Expression of Mig6 is driven via ERK1/2, constituting a physiologic negative feedback on EGFR activation ${ }^{44,45}$. Although not evaluated here, trametinib may serve to short-circuit this negative feedback by decreasing Mig6 and allowing unabated activation of alternate, apoptotic signaling downstream of EGFR such as STAT1.

In conclusion, our studies broadly illustrate the importance of understanding the cellular outcomes of cytoplasmic kinase inhibitors not only in terms of the pathway they are targeting, but also in terms of changes they insight to alternate signal transduction pathways induced from shared upstream receptors. These data support current clinical trials evaluating the efficacy of trametinib in the treatment of metastatic breast cancer (NCT02900664, NCT03065387). However, our results argue against the concurrent use of EGFR kinase inhibitors in these patients as this will block apoptotic, EGFR: STAT1 signaling, limiting the apoptotic effect of trametinib treatment. Current studies in the lab are exploring therapeutic approaches to enhance the antitumor effects of trametinib through specific augmentation of EGFR: STAT1 signaling.

\section{Acknowledgements}

The authors thank the members of the Wendt lab for critical reading of the manuscript. This research was supported in part by National Institutes of Health (R01CA207751) to M.K.W., the American Cancer Society (RSGCSM130259) and METavivor foundation to M.K.W., and Purdue Center for Cancer Research via an $\mathrm{NIH} \mathrm{NCl} \mathrm{grant} \mathrm{(P30CA023168).} \mathrm{The} \mathrm{authors} \mathrm{also}$ acknowledge the use of the facilities of the Bindley Bioscience Center, a core facility of the NIH-funded Indiana Clinical and Translational Sciences Institute.

Conflict of interest

The authors declare that they have no conflict of interest.

\section{Publisher's note}

Springer Nature remains neutral with regard to jurisdictional claims in published maps and institutional affiliations.

Supplementary Information accompanies this paper at (https://doi.org/ 10.1038/s41419-018-1034-7).

Received: 11 April 2018 Revised: 21 August 2018 Accepted: 5 September 2018

Published online: 24 September 2018

\section{References}

1. Steeg, P. S. Targeting metastasis. Nat. Rev. Cancer 16, 201-218 (2016).

2. Vignot, S., Besse, B., André, F., Spano, J.-P. \& Soria, J.-C. Discrepancies between primary tumor and metastasis: a literature review on clinically established biomarkers. Crit. Rev. Oncol. Hematol. 84, 301-313 (2012).

3. Cejalvo, J. M. et al. Intrinsic subtypes and gene expression profiles in primary and metastatic breast cancer. Cancer Res. 77, 2213-2221 (2017).

4. Niikura, N. et al. Loss of human epidermal growth factor receptor 2 (HER2) expression in metastatic sites of HER2-overexpressing primary breast tumors. J. Clin. Oncol. 30, 593-599 (2011).

5. Ali, R. \& Wendt, M. K. The paradoxical functions of EGFR during breast cancer progression. Signal Transduct. Target Ther. 2, 16042 (2017).

6. Choong, L.-Y. et al. Progressive loss of epidermal growth factor receptor in a subpopulation of breast cancers: implications in target-directed therapeutics. Mol. Cancer Ther. 6, 2828-2842 (2007).

7. Wendt, M. K., Smith, J. A. \& Schiemann, W. P. Transforming growth factor- $\beta-$ induced epithelial-mesenchymal transition facilitates epidermal growth factor-dependent breast cancer progression. Oncogene 29, 6485-6498 (2010). 
8. Wyckoff, J. et al. A paracrine loop between tumor cells and macrophages is required for tumor cell migration in mammary tumors. Cancer Res. 64 7022-7029 (2004)

9. Park, H. S. et al. High EGFR gene copy number predicts poor outcome in triple-negative breast cancer. Mod. Pathol. 27, 1212-1222 (2014).

10. Tischkowitz, M. et al. Use of immunohistochemical markers can refine prognosis in triple negative breast cancer. BMC Cancer 7, 134 (2007).

11. Jackson, N. M. \& Ceresa, B. P. Protein kinase G facilitates EGFR-mediated cell death in MDA-MB-468 cells. Exp. Cell Res. 346, 224-232 (2016).

12. Lim, Y. J., Jeon, S.-R., Koh, J. M. \& Wu, H.-G. Tumor growth suppression and enhanced radioresponse by an exogenous epidermal growth factor in mouse xenograft models with A431 Cells. Cancer Res. Treat. 47, 921-930 (2015).

13. Wendt, M. K. et al. The antitumorigenic function of EGFR in metastatic breast cancer is regulated by expression of Mig6. Neoplasia 17, 124-133 (2015).

14. Choi, J. et al. Epidermal growth factor induces cell death in the absence of overexpressed epidermal growth factor receptor and ErbB2 in various human cancer cell lines. Cancer Invest. 28, 505-514 (2010).

15. Grudinkin, P. S., Zenin, V. V., Kropotov, A. V., Dorosh, V. N. \& Nikolsky, N. N. EGFinduced apoptosis in A431 cells is dependent on STAT1, but not on STAT3. Eur. J. Cell Biol. 86, 591-603 (2007).

16. Andersen, P. et al. EGFR induces expression of IRF-1 via STAT1 and STAT3 activation leading to growth arrest of human cancer cells. Int. J. Cancer $\mathbf{1 2 2}$ 342-349 (2008).

17. Chin, Y. E., Kitagawa, M., Kuida, K., Flavell, R. A. \& Fu, X. Activation of the STAT signaling pathway can cause expression of caspase 1 and apoptosis. Mol. Cell. Biol. 17, 5328-5337 (1997)

18. Dickler, M. N. et al. A phase II trial of erlotinib in combination with bevacizumab in patients with metastatic breast cancer. Clin. Cancer Res. 14 7878-7883 (2008).

19. Dickler, M. N., Cobleigh, M. A., Miller, K. D., Klein, P. M. \& Winer, E. P. Efficacy and safety of erlotinib in patients with locally advanced or metastatic breast cancer. Breast Cancer Res. Treat. 115, 115-121 (2009).

20. von Minckwitz, G. et al. A multicentre phase II study on gefitinib in taxane- and anthracycline-pretreated metastatic breast cancer. Breast Cancer Res. Treat. 89, 165-172 (2005)

21. Sunakawa, Y. et al. Combined assessment of EGFR-related molecules to predict outcome of 1st-line cetuximab-containing chemotherapy for metastatic colorectal cancer. Cancer Biol. Ther. 17, 751-759 (2016).

22. Yardley, D. A. et al. Panitumumab, gemcitabine, and carboplatin as treatment for women with metastatic triple-negative breast cancer: a Sarah Cannon Research Institute phase II trial. Clin. Breast Cancer 16, 349-355 (2016).

23. Crozier, J. A. et al. N0436 (Alliance): a phase II trial of irinotecan with cetuximab in patients with metastatic breast cancer previously exposed to anthracycline and/or taxane-containing therapy. Clin. Breast Cancer $\mathbf{1 6}$ 23-30 (2016)

24. Trédan, O. et al. Ixabepilone alone or with cetuximab as first-line treatment for advanced/metastatic triple-negative breast cancer. Clin. Breast Cancer 15, 8-15 (2015).

25. Carey, L. A. et al. TBCRC 001: randomized phase II study of cetuximab in combination with carboplatin in stage IV triple-negative breast cancer. J. Clin. Oncol. 30, 2615-2623 (2012).

26. Smith, I. E. et al. A phase II placebo-controlled trial of neoadjuvant anastrozole alone or with gefitinib in early breast cancer. J. Clin. Oncol. 25, 3816-3822 (2007).
27. Gilmartin, A. G. et al. GSK1120212 (JTP-74057) is an inhibitor of MEK activity and activation with favorable pharmacokinetic properties for sustained in vivo pathway inhibition. Clin. Cancer Res. 17, 989-1000 (2011).

28. Wendt, M. K. Taylor, M. A, Schiemann, B. J., Sossey-Alaoui, K. \& Schiemann, W. $P$. Fibroblast growth factor receptor splice variants are stable markers of oncogenic transforming growth factor $\beta 1$ signaling in metastatic breast cancers. Breast Cancer Res. 16, R24 (2014).

29. Balanis, N. et al. Epithelial to mesenchymal transition promotes breast cancer progression via a fibronectin-dependent STAT3 signaling pathway. J. Biol. Chem. 288, 17954-17967 (2013).

30. Balanis, N., Yoshigi, M., Wendt, M. K., Schiemann, W. P. \& Carlin, C. R. $\beta 3$ integrin-EGF receptor cross-talk activates p190RhoGAP in mouse mammary gland epithelial cells. Mol. Biol. Cell 22, 4288-4301 (2011).

31. Strickland, L. B., Dawson, P. J., Santner, S. J. \& Miller, F. R. Progression of premalignant MCF10AT generates heterogeneous malignant variants with characteristic histologic types and immunohistochemical markers. Breast Cancer Res. Treat. 64, 235-240 (2000).

32. Bartolowits, M. D. et al. Selective inhibition of STAT3 phosphorylation using a nuclear-targeted kinase inhibitor. ACS Chem. Biol. 12, 2371-2378 (2017).

33. Cheon, H. \& Stark, G. R. Unphosphorylated STAT1 prolongs the expression of interferon-induced immune regulatory genes. Proc. Natl. Acad. Sci. USA. 106 9373-9378 (2009)

34. Kil, S. J. \& Carlin, C. EGF receptor residues Leu679, Leu680 mediate selective sorting of ligand-receptor complexes in early endosomal compartments. J. Cell. Physiol. 185, 47-60 (2000).

35. Högnason, T. et al. Epidermal growth factor receptor induced apoptosis: potentiation by inhibition of Ras signaling. FEBS Lett. 491, 9-15 (2001).

36. Prasad, K. A. \& Church, J. G. EGF-dependent growth inhibition in MDA-468 human breast cancer cells is characterized by late $\mathrm{G} 1$ arrest and altered gene expression. Exp. Cell Res. 195, 20-26 (1991).

37. Liu, J. J., Horst, R., Katritch, V., Stevens, R. C. \& Wüthrich, K. Biased signaling pathways in $\beta 2$-adrenergic receptor characterized by 19F-NMR. Science $\mathbf{3 3 5}$ 1106-1110 (2012).

38. Knudsen, S. L. J., Mac, A. S. W., Henriksen, L., van Deurs, B. \& Grøvdal, L. M. EGFR signaling patterns are regulated by its different ligands. Growth Factors 32 155-163 (2014).

39. Chakraborty, S. et al. Constitutive and ligand-induced EGFR signalling triggers distinct and mutually exclusive downstream signalling networks. Nat. Commun. 5, 5811 (2014)

40. Brand, T. M. et al. Nuclear epidermal growth factor receptor is a functional molecular target in triple-negative breast cancer. Mol. Cancer Ther. 13 1356-1368 (2014).

41. Brand, T. M., lida, M., Li, C. \& Wheeler, D. L. The nuclear epidermal growth factor receptor signaling network and its role in cancer. Discov. Med. 12, 419-432 (2011).

42. Wang, Y.-N. et al. COPI-mediated retrograde trafficking from the Golgi to the ER regulates EGFR nuclear transport. Biochem. Biophys. Res. Commun. 399, 498-504 (2010).

43. Denais, C. M. et al. Nuclear envelope rupture and repair during cancer cell migration. Science 352, 353-358 (2016).

44. Keeton, A. B., Xu, J., Franklin, J. L. \& Messina, J. L. Regulation of Gene33 expression by insulin requires MEK-ERK activation. Biochim. Biophys. Acta 1679 248-255 (2004).

45. Fiorini, M. et al. Expression of RALT, a feedback inhibitor of ErbB receptors, is subjected to an integrated transcriptional and post-translational control. Oncogene 21, 6530-6539 (2002). 